\title{
Measurement and simulation of transverse Schottky noise with space charge
}

\author{
O. Boine-Frankenheim, V. Kornilov, and S. Paret \\ Gesellschaft für Schwerionenforschung (GSI) mbH, Planckstraße 1, D-64291 Darmstadt, Germany
}

(Received 5 June 2008; published 17 July 2008)

\begin{abstract}
The effect of moderate space charge on the transverse Schottky spectrum of a coasting beam is studied using measurements and simulations together with an analytic model. The measurements of transverse Schottky bands from heavy ion beams were performed in the SIS-18 synchrotron at GSI. In addition, we analyze the noise spectrum from a particle tracking code with self-consistent space charge. Both results are compared to an analytic model that is based on the dispersion relation for linear space charge forces and chromatic betatron tune spreads. The analytic model reproduces the characteristic deformation of Schottky bands with increasing space charge, observed in both measurement and simulation.
\end{abstract}

DOI: 10.1103/PhysRevSTAB.11.074202

PACS numbers: 29.20.D-, 29.27.Bd

\section{INTRODUCTION}

Transverse space charge plays an important role in the beam stability considerations for high intensity synchrotrons and storage rings. Although the incoherent space charge force alone does not cause coherent instabilities, it can modify or even remove Landau damping of transverse dipole oscillations [1,2]. The role of space charge is especially important for the envisaged operation with high quality and high intensity ion beams in the FAIR synchrotrons [3].

During machine operation with stable beams it is highly desirable to be able to directly measure space charge effects, like the induced betatron tune shift and the modified Landau damping rate.

A possible method is to measure the transverse beam transfer function with space charge (see e.g. [4]). This requires to excite dipole oscillations using, e.g., an external noise source. Alternatively, one can measure the "natural" fluctuation spectrum of dipole oscillations at a pickup probe. The Schottky noise spectrum, divided into separate bands, contains information on the incoherent and coherent frequencies in the beam [5].

In the longitudinal plane space charge results in the wellknown double-peaked shape of Schottky bands from coasting beams [6]. This characteristic deformation has been observed in many cooler storage rings (see e.g. [4]). From the deformation the longitudinal space charge impedance and the stability properties of the beam can be deduced.

With regard to transverse Schottky noise it has been suggested in Ref. [7] to use the spectrum of coherent dipole oscillations in order to determine the incoherent space charge tune shift. The modification of Schottky bands with space charge has been calculated in Refs. [7,8] from the linearized Vlasov equation including the nonlinear components of the space charge force for a Gaussian beam profile. The nonlinear components are especially important for strong space charge (see e.g. [9]).
Understanding the space charge induced modification of the transverse Schottky spectrum is an important issue for beam diagnostics in high intensity machines. The Schottky spectrum is used routinely to probe, e.g., machine tunes, the tune spreads, or the emittance evolution. Any modification of the spectrum should be known in advance in order to extract information from a specific Schottky band.

The present study focuses on the deformation of transverse Schottky bands by space charge in coasting beams and for a chromatic tune spread. The strength of the space charge effect is usually described in terms of the ratio of the space charge tune shift to the chromatic tune spread. Here we report about transverse Schottky measurements performed with heavy ion beams in the SIS-18 synchrotron [10] for moderate space charge.

In addition, we will discuss results of studies in which we use the numerical noise of the particle tracking code PATRIC [11] in order to simulate the Schottky noise from the real beam. Similar simulation studies have been performed for the longitudinal Schottky noise from bunched beams $[12,13]$. Here we will present results for the numerical fluctuation spectrum of coherent dipole oscillations including self-consistent space charge. In the simulations we chose beam parameters close to the SIS-18 experiment.

The noise spectra obtained from the measurements as well as from the simulations are compared to an analytic model that is based on the dispersion relation for linear space charge forces and chromatic betatron tune spreads. The dispersion relation for transverse dipole oscillations with linear space charge has been discussed, e.g., in Refs. [14,15]. A dispersion relation including the nonlinear components of the space charge force has been presented in Ref. [1]. For weak or moderate space charge, one can justify to omit the nonlinear components.

The paper is organized as follows: In Sec. II we describe the analytic model. In Sec. III the results of simulation studies are presented and compared to the analytic model. The measurements are discussed in Sec. IV. The conclusions are in Sec. V. 


\section{TRANSVERSE SCHOTTKY SPECTRUM AND COLLECTIVE EFFECTS}

The transverse Schottky signal of the beam, detected by a pickup (PU) probe, is given by the transverse beam offset times current

$$
d(t)=\sum_{j=1}^{N} x_{j}(t) I_{j}(t)
$$

with the offset $x_{j}(t)$ and the current $I_{j}(t)$ of the $j$ th particle at the position of the PU in the ring (see, e.g., Ref. [5], page 14). The sum extends over all $N$ particles in the beam. The Schottky noise power spectrum as a function of the frequency $\Omega$ is defined as

$$
P(\Omega)=|d(\Omega)|^{2}
$$

with the Fourier transformed PU signal $d(\Omega)$. At low beam intensities the spectrum $P^{0}(\Omega)$ consists of bands centered at frequencies

$$
\Omega_{n}=(n \pm Q) \omega_{0}
$$

with the harmonic number $n$, the tune $Q$, and the revolution frequency $\omega_{0}$. The form of each band $P_{n}^{0}$ reflects the incoherent betatron frequency distribution

$$
P_{n}^{0}(\Omega) \propto g\left(\frac{\Omega_{n}-\Omega}{\delta \omega}\right)
$$

with the incoherent frequency distribution $g$ and the rms frequency spread $\delta \omega=\omega_{0} \delta Q$. For chromatic betatron frequency spreads $g(\delta)$ is determined by the distribution of the longitudinal momentum deviation $\delta$. The effective chromaticity for upper and lower sidebands $(n \pm Q)$ is

$$
S_{ \pm}=\left[ \pm \xi-\eta_{0}(n \pm Q)\right]
$$

with the frequency slip factor $\eta_{0}=1 / \gamma_{t}^{2}-1 / \gamma_{0}^{2}$, the transition parameter $\gamma_{t}$, and the chromaticity $\xi$. In the following, for simplicity, only upper sidebands will be considered with $S=S_{+}$. The resulting rms tune spread is

$$
\delta Q_{\xi}=S \delta_{\mathrm{rms}}
$$

with the rms momentum spread $\delta_{\text {rms }}$.

\section{A. Collective effects and the dispersion relation}

At high beam intensities coherent and incoherent tune shifts induced by space charge and by image currents in the pipe are of relevance. For a coasting beam the real coherent betatron tune shift $\Delta Q^{\text {coh }}=\Delta \Omega / \omega_{0}$ induced by an ideal conducting wall is (see e.g. [15], page 360)

$$
\Delta Q^{\mathrm{coh}}=-i \frac{q I R}{4 \pi \beta_{0} E_{0} Q_{0}} Z_{\perp}
$$

with the imaginary transverse impedance

$$
Z_{\perp}=-i \frac{Z_{0} R}{\beta_{0}^{2} \gamma_{0}^{2} b^{2}},
$$

the bare tune $Q_{0}$, the energy $E_{0}$, the pipe radius $b$, the vacuum impedance $Z_{0}=377 \Omega$, the ring radius $R$, and the relativistic parameters $\beta_{0}$ and $\gamma_{0}$. The incoherent space charge tune shift in a round beam and for a homogeneous density (Kapchinski-Vladimirski or KV distribution) is determined through (see e.g. [16])

$$
\Delta Q^{\mathrm{sc}}=-\frac{q I R}{4 \pi \varepsilon_{0} c E_{0} \beta_{0}^{3} \gamma_{0}^{2} \epsilon} .
$$

For a homogenous beam density, all particles experience the same space charge tune shift. For a Gaussian profile the space charge force is nonlinear and the tune shift depends on the betatron amplitude. The resulting tune spread is $\delta Q^{\mathrm{sc}} \approx 0.5\left|\Delta Q^{\mathrm{sc}}\right|$, if the Gaussian distribution is truncated at $2 \sigma$ (Ref. [15], page 105). For strong space charge the modification of the Schottky signal due to the nonlinear self-force has been analyzed in Ref. [7]. Within our simplified analytic model, we assume a constant space charge tune shift $\Delta Q^{\text {sc }}$ defined for the rms equivalent $\mathrm{KV}$ distribution. Thereby we neglect the space charge induced tune spread. This can be justified for moderate space charge and $\delta Q^{\text {sc }} \lesssim \delta Q_{\xi}$, which is the case in the frequency range and for the beam parameters for which Schottky measurements can be performed in the SIS- 18 and also in the ESR storage ring at GSI. For constant tune shifts the dispersion relation $D(z)=1$ for coasting beam dipole modes can be obtained from (see e.g. [14])

$$
D(z)=\frac{\Delta Q^{\mathrm{coh}}-\Delta Q^{\mathrm{sc}}}{S \delta_{\mathrm{rms}}} \int_{-\infty}^{\infty} \frac{g(\delta) d \delta}{z-\delta}
$$

with the normalized frequency

$$
z=\frac{\Omega-n \omega_{0}-\omega_{\beta}}{S \delta_{\mathrm{rms}} \omega_{0}}
$$

and the incoherent betatron frequency

$$
\omega_{\beta}=\omega_{\beta, 0}+\Delta \omega^{\mathrm{sc}} .
$$

$\omega_{\beta, 0}=\omega_{0} Q_{0}$ is the bare betatron frequency, $\Delta \omega^{\mathrm{sc}}=$ $\omega_{0} \Delta Q^{\text {sc }}$ is the incoherent frequency shift due to space charge.

The normalized frequency $z$ is corrected for the incoherent space charge shift $\Delta \omega^{\mathrm{sc}}$, but not for the coherent shift. This has the advantage that coherent and incoherent shifts can both be represented by the same $D(z)$. The difference between coherent and incoherent effects is contained entirely in $z$. For a beam with a Gaussian momentum distribution, the dispersion function can be written as

$$
D(z)=-i \sqrt{\frac{2}{\pi}}\left(U_{\mathrm{coh}}-U_{\mathrm{sc}}\right) w(z / \sqrt{2})
$$

with the complex error function 


$$
w(z)=\exp \left(-z^{2}\right)[1-\operatorname{erf}(-i z)],
$$

the impedance parameter

$$
U_{\mathrm{coh}}=-\frac{\Delta Q^{\mathrm{coh}}}{S \delta_{\mathrm{rms}}},
$$

and the space charge parameter

$$
U_{\mathrm{sc}}=-\frac{\Delta Q^{\mathrm{sc}}}{S \delta_{\mathrm{rms}}}
$$

\section{B. Modified Schottky spectrum}

Following the approaches in Refs. [5,7], we may write the power spectrum for a Schottky band including collective effects as

$$
P(\Omega)=\frac{P^{0}(z)}{|\epsilon(z)|^{2}},
$$

where the dielectric function $\epsilon(z)$ is given by

$$
\epsilon(z)=1-D(z),
$$

$z$ is the shifted, normalized frequency, see Eq. (11), and $P^{0}(z) \propto g(z)$ is the low intensity spectrum. In Ref. [5], the dielectric function $\epsilon$ is discussed in terms of the beam transfer function, which is equivalent to our description. In our formulation the dispersion function $D(z)$ from Eq. (13) in $\epsilon(z)$ accounts for incoherent and coherent effects.

Figure 1 shows $P(z)$ from Eq. (17) for different space charge parameters $U_{\mathrm{sc}}$ and $U_{\mathrm{coh}}=0$. The characteristic distortion of the spectrum with increasing space charge is clearly visible. For finite $U_{\mathrm{coh}}$ and $U_{\mathrm{sc}}=0$ the curves are identical if one plots $P(-z)$. In Fig. 2 we plot $P(\Omega)$ for the same space charge parameters, but as a function of the "physical" relative frequency $\Omega-\Omega_{+}$with the upper

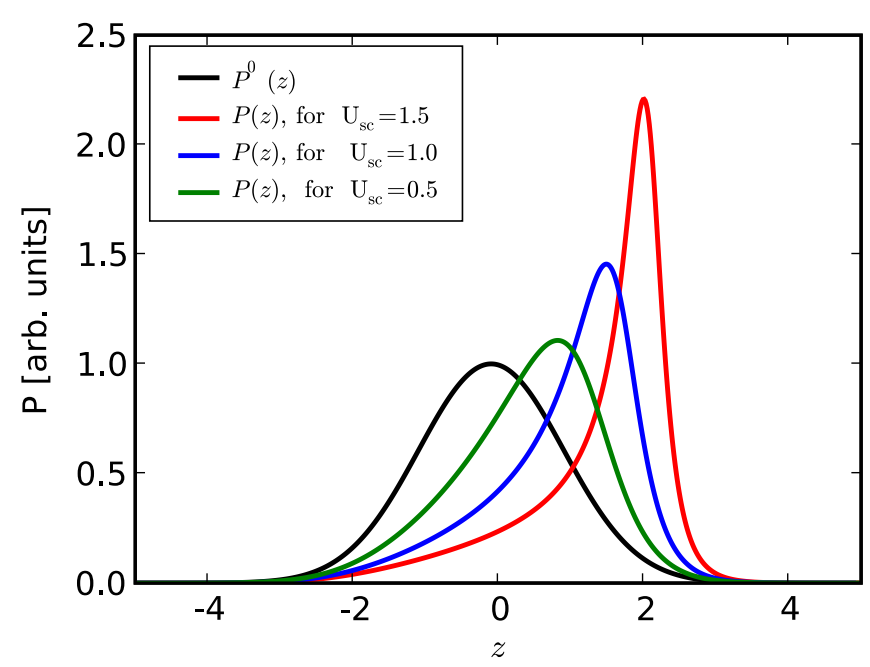

FIG. 1. (Color) $P(z)$ from Eq. (17) for an upper sideband and for different $U_{\mathrm{sc}}$ with $U_{\mathrm{coh}}=0$.

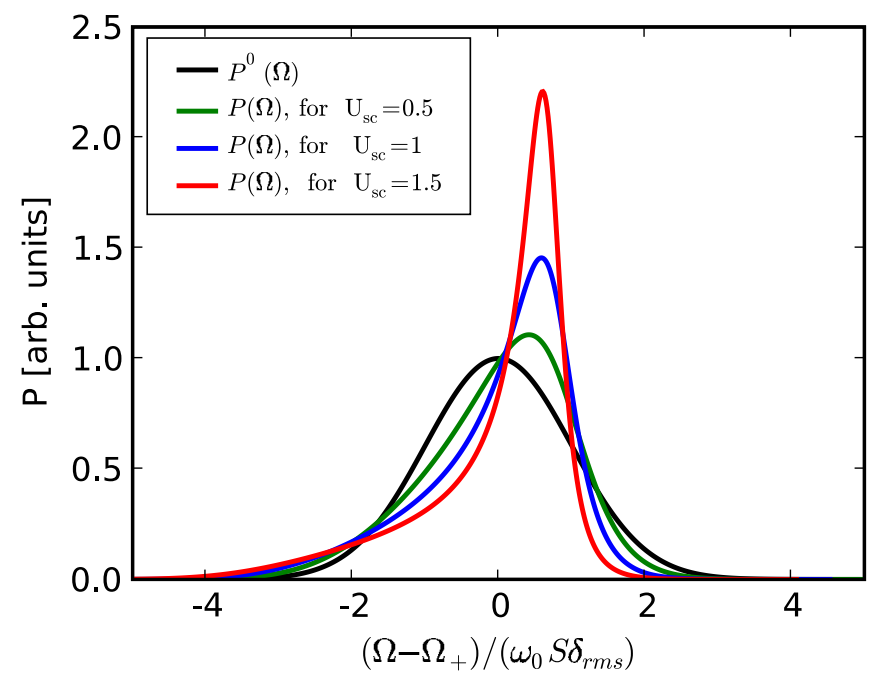

FIG. 2. (Color) $P(\Omega)$ from Eq. (17) for an upper sideband and for different $U_{\mathrm{sc}}$ with $U_{\mathrm{coh}}=0$.

sideband frequency $\Omega_{+}=\left(n+Q_{0}\right) \omega_{0}$. With increasing space charge parameter, the center of the Schottky band remains close to the original center of the undisturbed band at $\Omega_{+}$. The difference between the effect of space charge and the effect of an imaginary impedance as a function of $\Omega$ is presented in Fig. 3. Here we compare $P(\Omega)$ for $U_{\text {sc }}=$ 1 and $U_{\text {coh }}=0$ with the result for $U_{\mathrm{sc}}=0$ and $U_{\text {coh }}=1$, again as a function of the relative frequency $\Omega-\Omega_{+}$. For finite $U_{\text {coh }}$ the center of the Schottky band shifts towards the coherent tune $\Omega_{+}+\Delta \Omega$ indicated as a dotted green line in Fig. 3. The observation that the Schottky band with space charge remains close to $\Omega_{ \pm}$and does not move towards the shifted incoherent frequency can be explained by the fact that the incoherent space charge force acts

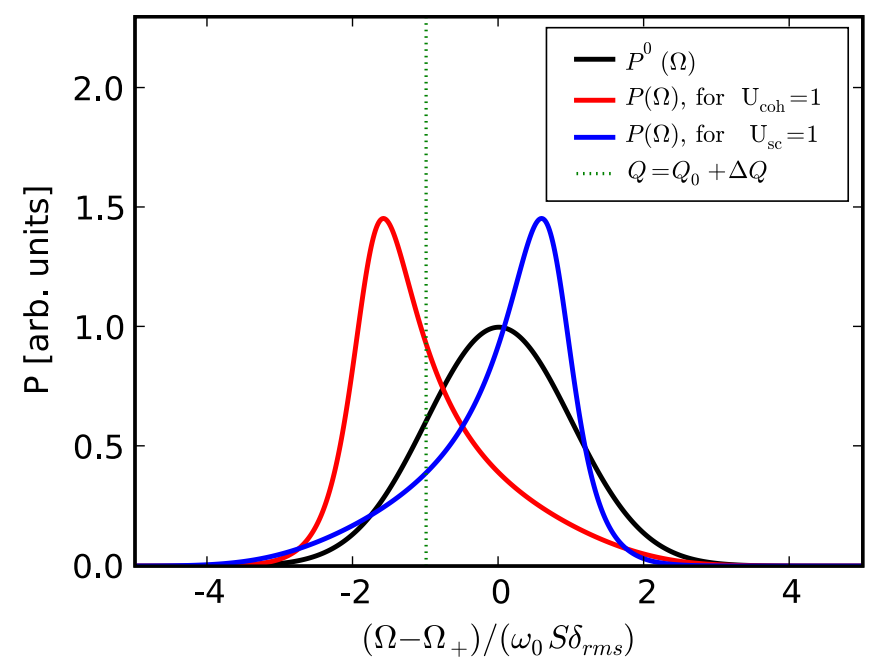

FIG. 3. (Color) $P(\Omega)$ from Eq. (17) for an upper sideband and for $U_{\text {coh }}=U_{\text {sc }}=0$ (black curve), $U_{\text {sc }}=1$ and $U_{\text {coh }}=0$ (blue curve), as well as $U_{\mathrm{sc}}=0$ and $U_{\text {coh }}=1$ (red curve). The dotted vertical line indicates the shifted incoherent and coherent tune. 
relative to the beam center on individual particles. It does not change the coherent frequency of beam center oscillations. The coherent image current force, on the contrary, acts directly on the bunch center and therefore it shifts the Schottky band. An important example is the hypothetical case where coherent and incoherent forces act simultaneously and equally strong, $\Delta \Omega=\Delta \omega^{\mathrm{sc}}$. This results in $D(z)=0$ and

$$
P(\Omega)=P^{0}(z) .
$$

Thus, the modified Schottky spectrum for $U_{\mathrm{sc}}=U_{\mathrm{coh}}$ within our analytic model corresponds to the low intensity spectrum $P^{0}(\Omega)$, but shifted by $\Delta \omega^{\mathrm{sc}}=\Delta \Omega$. It is impossible to verify this conclusion in an experiment, but we will study this case in our numerical simulations in the next section.

\section{SIMULATION SCHEME AND RESULTS}

In this section we will discuss results of studies in which we use the numerical noise of the particle tracking code PATRIC [11] in order to simulate the Schottky noise from the real beam. Together with the experiment the simulations should further confirm the applicability of the simplified analytic model presented in Sec. II. Using the simulations we can explore the Schottky noise spectrum for a wider range of space charge, impedance, and machine parameters as compared to the experiments. Furthermore the simulation can be used to study the modification of Schottky signals due to additional effects, like intrabeamscattering and octupoles.

The transverse noise signal is obtained from the simulation code via

$$
d(t)=\sum_{j=1}^{N_{p}} x_{j}(t) I_{j}(t)
$$

at a fixed PU position in the ring. Here $N_{p} \ll N$ is the number of macroparticles in the simulation, $x_{j}(t)$ is the offset, and $I_{j}(t)$ is the current of the $j$ th macroparticle at the location of the PU. Figure 4 shows the resulting noise power spectrum together with the tune. The lines appear at frequencies $f_{n, \pm}=f_{0}\left(n \pm Q_{0}\right)$, with the bare tune $Q_{0}$ and the revolution frequency $f_{0}$. For simplicity we use a round beam and equal tunes in the simulation study. In the simulation study we focus on the power spectrum of the $n=0$ noise band. The $n=0$ mode represents fixed offset oscillations with no phase difference along the ring circumference. Therefore a two-dimensional (2D) solver for the space charge field can be employed in the simulations. Focusing on the $n=0$ band reduces also the required number of macroparticles $N_{p}$. On the one hand, the finite number of macroparticle is the source of the numerical noise. On the other hand, the number of macroparticles has to be large enough to resolve the mode structure and to reduce the artificial collision rate (see e.g. [17]). In the

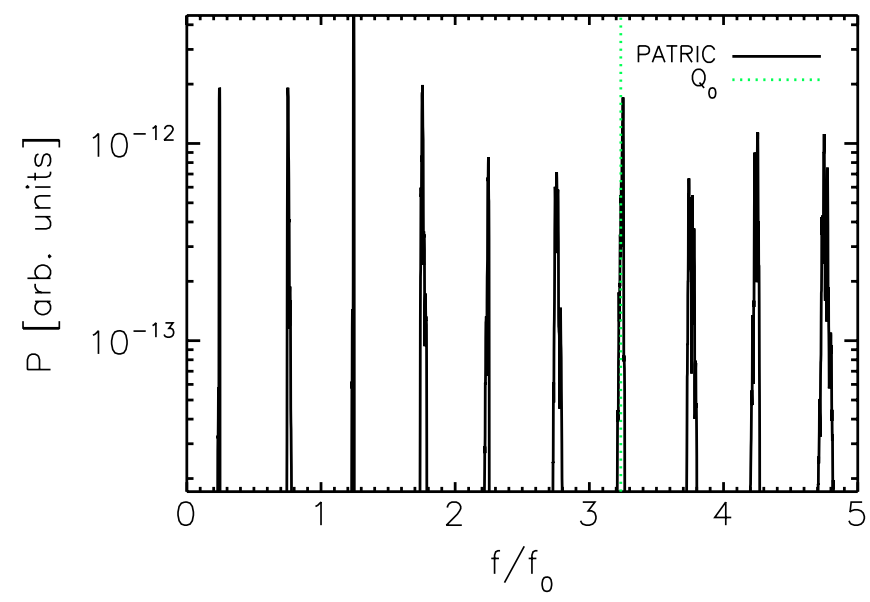

FIG. 4. Noise power spectrum obtained from the simulation code. The dotted, green curve indicates the position of the bare tune $Q_{0}$.

simulation $N_{p}=10^{6}$ macroparticles were initially loaded in $\left(x, y, x^{\prime}, y^{\prime}\right)$ trace space to reproduce a transverse waterbag distribution. We also studied transverse Gaussian distributions, but for the parameter regime under consideration the obtained simulation noise spectra showed no noticeable differences. The momentum distribution is chosen as a Gaussian and the macroparticles are distributed homogeneously around the ring. Afterwards the fluctuation of the beam offset is recorded over a time interval of $T_{s} \gtrless$ $1000 /\left(Q_{0} f_{0}\right)$. A 2 D Poisson solver on a Cartesian grid with rectangular boundaries is employed. The distance between the beam and the wall boundaries is chosen sufficiently large in order not to modify the results. The coherent tune shift caused by image currents in the pipe can be added independently on the Poisson solver via impedance kicks [9].

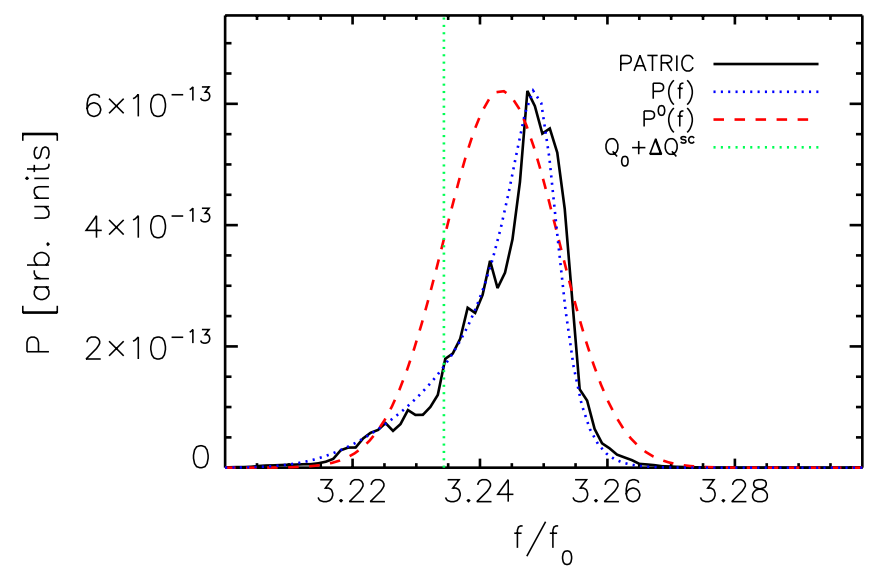

FIG. 5. (Color) Noise spectrum from the PATRIC simulation with self-consistent space charge. The space charge parameter is $U_{\mathrm{sc}}=1$. The dashed red curve represents the noise spectrum for $U_{\mathrm{sc}}=0$ with its maximum adjusted to the $U_{\mathrm{sc}}=1$ curve. The vertical green line represents the shifted tune $Q=Q_{0}+$ $\Delta Q^{\mathrm{sc}}$. 


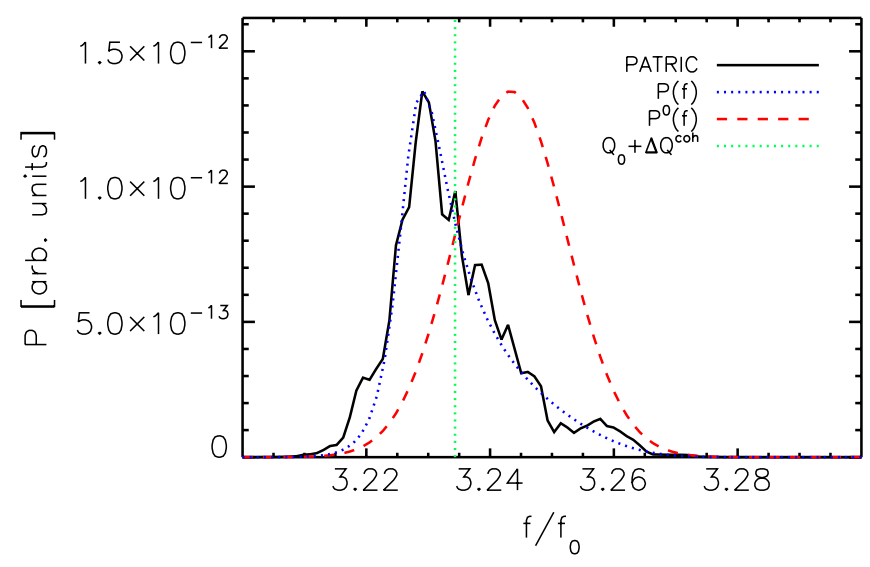

FIG. 6. (Color) Schottky spectrum from the PATRIC simulation with a constant imaginary impedance corresponding to $U_{\text {coh }}=$ 1. The dashed red curve represents the noise spectrum for $U_{\text {coh }}=0$ with its maximum adjusted to the $U_{\text {coh }}=1$ curve. The vertical green line represents the shifted coherent tune $Q^{\mathrm{coh}}=Q_{0}+\Delta Q^{\mathrm{coh}}$.

The simulations are performed for beam parameters corresponding to $\left|\Delta Q^{\mathrm{sc}}\right|=\delta Q_{\xi}$ or $U_{\mathrm{sc}}=1$. This parameter regime is accessible in Schottky measurements in the SIS-18 (see Sec. IV). In order to further speed up the simulations, we increased the rms momentum spread together with the beam current by a factor of 5 relative to the typical SIS- 18 beam parameters, keeping the space charge parameter $U_{\text {sc }}$ constant.

Figure 5 shows the simulation noise power spectrum obtained for $U_{\mathrm{sc}}=1$ together with the analytic result $P(z)$ from Eq. (17) for $n=0$. The maximum of $P(z)$ is fitted to the simulation result. It can be seen that the shape of the noise spectrum obtained from the simulation is reproduced very well by the analytic approach. In Fig. 6

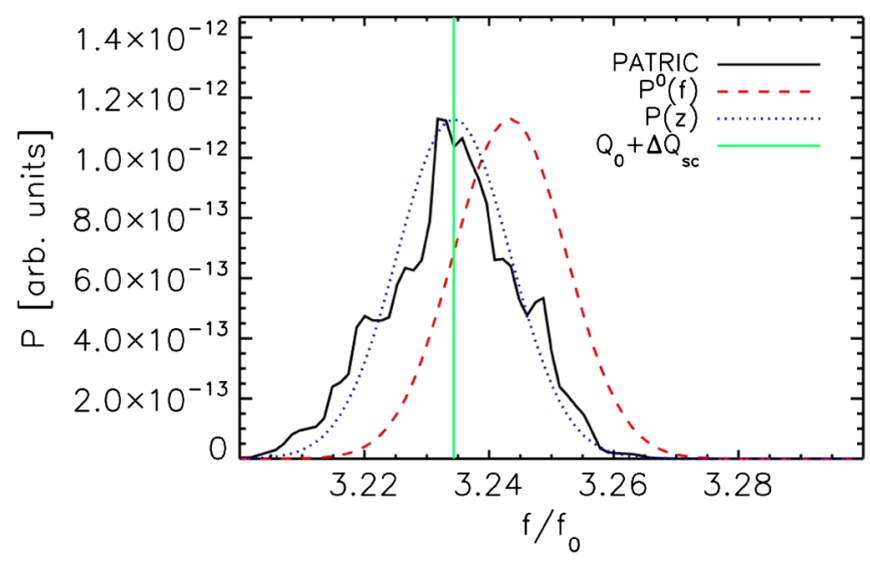

FIG. 7. (Color) Schottky spectrum from the PATRIC simulation with a constant imaginary impedance and space charge. The impedance and space charge parameters are $U_{\mathrm{coh}}=U_{\mathrm{sc}}=1$. The dashed red curve represents the noise spectrum for $U_{\mathrm{sc}}=$ $U_{\text {coh }}=0$. The vertical green line represents the shifted tune $Q^{\text {coh,inc }}=Q_{0}+\Delta Q$. the simulation result is plotted for $U_{\mathrm{sc}}=0$ and $U_{\mathrm{coh}}=1$. Also in this case a good agreement between Eq. (17) with $U_{\text {coh }}=1$ and the shape of the simulation noise spectrum can be observed. Finally, Fig. 7 shows the simulation result for $U_{\mathrm{sc}}=U_{\mathrm{coh}}=1$. In agreement with the analytical model, the spectrum obtained from the simulation can be well described by the shifted Gaussian distribution $P^{0}(z)$ centered at the coherent tune $Q^{\text {coh }}$. Our studies predict a good agreement between the simulations and expression Eq. (17) for space charge and impedance parameters in the range $\{0,2\}$.

\section{MEASUREMENTS AND RESULTS}

Schottky measurements were performed in the SIS-18 [10] with coasting ${ }^{40} \mathrm{Ar}^{18+}$ beams at injection energy $(11.4 \mathrm{MeV} / \mathrm{u})$ and for $N \approx 7 \times 10^{9}$ ions. The beam profiles were measured using a ionization profile monitor [18]. The rms momentum spread was obtained from the rms width of a longitudinal Schottky band. From the rms beam radii of the Gaussian profiles we obtained the emittances and the corresponding space charge tune shifts (see Table I for the relevant beam parameters). The emittance of the rms equivalent $\mathrm{KV}$ distribution is used ( $2 \sigma$ beam radius). The elliptical cross section of the beam was taken into account by substituting the vertical emittance $\epsilon_{\mathrm{v}}$ in Eq. (9) by $\left(\epsilon_{\mathrm{v}}+\sqrt{\epsilon_{\mathrm{v}} \epsilon_{\mathrm{h}} Q_{\mathrm{v}} / Q_{\mathrm{h}}}\right) / 2$, where $\epsilon_{\mathrm{h}}$ is the horizontal emittance. For the interpretation of the measurements, the coherent tune shift due to the image currents in the wall could be ignored relatively to the direct space charge effect because in the experiments the beam radii were much smaller than the pipe radii. The vertical Schottky signals were obtained from the dedicated Schottky PU in the SIS18. In the vertical direction the PU plates are closer to the beam and therefore the signal to noise ratio is higher. A real-time spectrum analyzer was employed to analyze and to store the Schottky spectra.

A pair of lower and upper vertical sidebands measured around the $m=75$ th harmonic $\left(m=f / f_{0}\right)$ is shown in Figs. 8 and 9, respectively. Clearly observable is the asym-

TABLE I. Beam and machine parameters during the measurement. The space charge tune shifts are evaluated from the measured emittances.

\begin{tabular}{lc}
\hline \hline Ion & ${ }^{40} \mathrm{Ar}^{18+}$ \\
$N$ & $7 \times 10^{9}$ \\
Energy & $11.4 \mathrm{MeV} / \mathrm{u}$ \\
$f_{0}$ & $213.1 \mathrm{kHz}$ \\
$\delta_{\text {rms }}$ & $6.6 \times 10^{-4}$ \\
$\epsilon_{\text {ver }}^{\mathrm{rms}}$ & $4.6 \mathrm{~mm} \mathrm{mrad}$ \\
$\epsilon_{\text {hor }}^{\mathrm{rms}}$ & $5.7 \mathrm{~mm} \mathrm{mrad}$ \\
$Q_{\text {hor }}$ & 4.21 \\
$Q_{\text {ver }}$ & 3.31 \\
$\Delta Q_{x}^{\text {sc }}$ & -0.02 \\
$\Delta Q_{y}^{\text {sc }}$ & -0.03 \\
\hline \hline
\end{tabular}




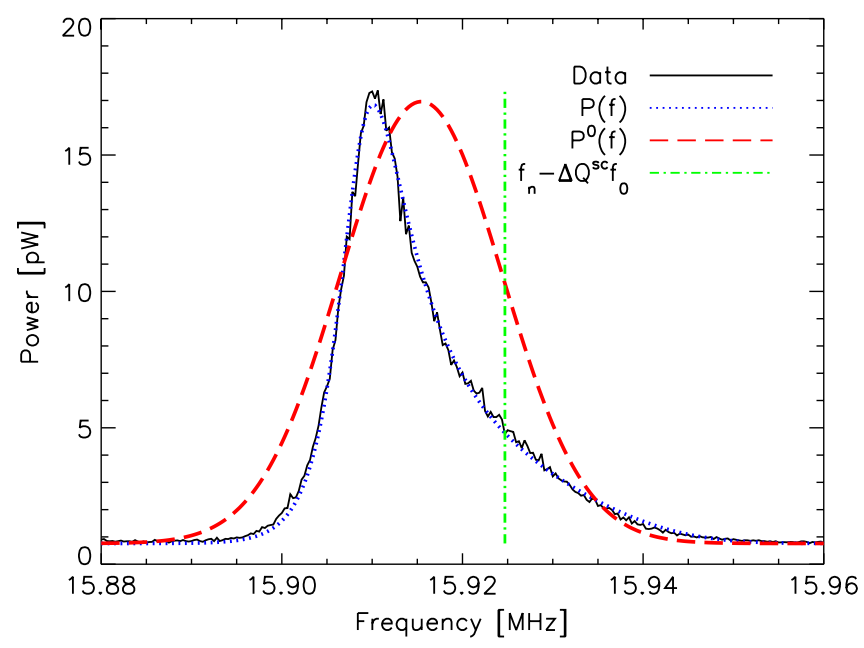

FIG. 8. (Color) Lower vertical sideband of the 75th harmonic measured in SIS-18. The measured data is plotted as a black curve. The dotted blue curve represents the analytic expression Eq. (17) with fitted parameters. The dashed red curve shows the expected Schottky spectrum for $U_{\mathrm{sc}}=0$ (maximum adjusted). The vertical green line indicates the incoherent frequency $f_{n, \pm} \pm \Delta Q^{\mathrm{sc}} f_{0}$ shifted by space charge.

metry of the bands which we refer to the effect of space charge. In both figures the measured sidebands are compared with the analytic model from Eq. (17). Here $P(f)$ as a function of the frequency $f=\Omega / 2 \pi$ is the result of the adaptation of Eq. (17) to the data using the peak value, $\delta_{\text {rms }}, \omega_{\beta}$ and the space charge parameter $U_{\text {sc }}$ as fit parameters. In addition $P^{0}(f)$ in Figs. 8 and 9 show the expected sidebands without space charge effects. For the lower sideband (Fig. 8) the analytic model $P(f)$ matches very well the measured data. The upper sideband in Fig. 9 is still within a reasonable agreement.

The space charge parameters obtained from the fit are $U_{\mathrm{sc}}=1.28$ for the upper sideband and 1.03 for the lower

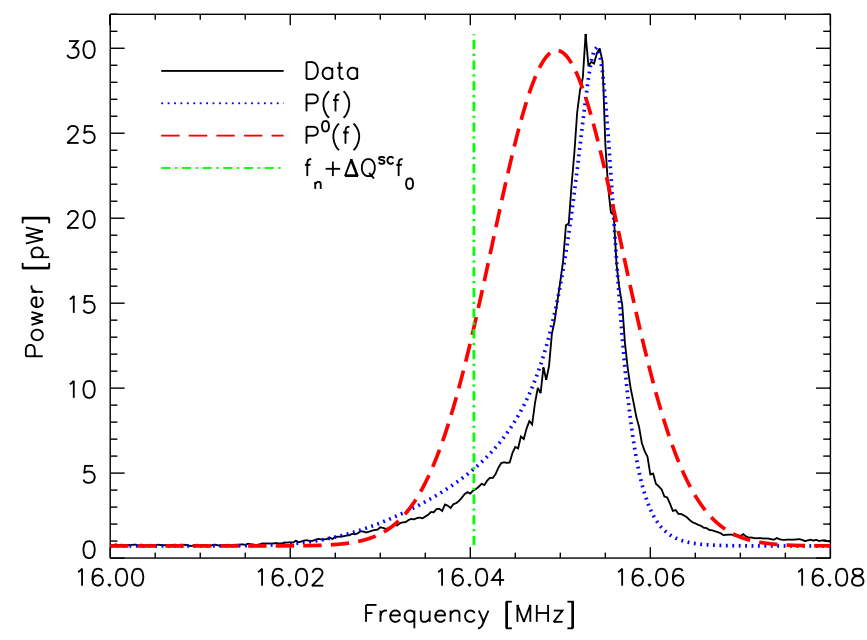

FIG. 9. (Color) Upper vertical sideband of the 75th harmonic measured in SIS-18. The meaning of the curves is the same as in Fig. 8. one. The difference in the space charge parameters arises because of the different $S_{ \pm}$for the two bands. The resulting vertical tune shift is $\Delta Q_{\text {fit }}^{\text {sc }} \approx-0.04$ which is close to $\Delta Q_{y}^{\mathrm{sc}}=-0.03$ estimated from the beam parameters.

\section{CONCLUSIONS}

In summary, an analytic model Eq. (17) for the Schottky noise spectrum from a coasting beam affected by moderate space charge and image currents is described. Within this model space charge or image currents cause characteristic deformations of the shape of a band. The incoherent space charge force does not shift the center of the band relative to the bare tune. In the presence of image currents the band is shifted towards the coherent tune.

We successfully compared the analytic model with selfconsistent simulations and with experiments performed in the SIS-18 synchrotron. The noise spectrum obtained from a particle tracking code showed a reasonably good agreement with the analytic model for moderate space charge. However, one has to keep in mind that the analysis of the noise spectrum of particle codes represents a complex "numerical experiment" with possible artificial effects, e.g., due to the finite particle number, the required interpolations on a finite grid and the finite time step. Here we used the simulations to further validate our analytic approach. On the other hand, our work suggests to use analytic models for the Schottky noise to validate particle codes with self-consistent space charge solvers.

In measurements of the transverse Schottky signals performed in the SIS-18 synchrotron, we clearly observed the deformation of bands caused by space charge. The analytic model was fitted to the measured spectra. From the fit we determined the space charge tune shift $\Delta Q^{\text {sc }}$ and the space charge parameter $U_{\mathrm{sc}}$. The results were found in good agreement with the values estimated independently from the measured beam parameters.

In conclusion, we showed that for moderate space charge an analytic model based on linear forces can adequately describe the transverse Schottky noise spectrum of coasting beams. The analytic expression can be used, e.g., to determine the transverse space charge tune shift from measured Schottky signals. If space charge effects are important also the measurement of the machine tune and of the tune spread from the Schottky signal require a fit to the analytic model. Both quantities cannot be obtained directly from the distance and from the width of different Schottky bands, as it is usually done for low beam intensities.

\section{ACKNOWLEDGMENTS}

The authors acknowledge the support of the European Community Research Infrastructures Action under the FP6 program: Structuring the European Research AreaSpecific Support Action-Design Study (Contract No. 515873 -DIRACsecondary-Beams). 
[1] D. Möhl and H. Schönauer, Proceedings of the IX International Conference on High Energy Accelerators, Stanford, 1974, p. 380.

[2] K. Y. Ng, AIP Conf. Proc. 773, 365 (2005).

[3] O. Boine-Frankenheim, I. Hofmann, and V. Kornilov, Proceedings of EPAC 2006, Edinburgh, UK, 2006, p. 1882.

[4] U. Schaaf, K. Beckert, D. Budicin, H. Eickhoff, B. Franzke, I. Hofmann, G. Kalisch, F. Nolden, P. Spaedtke, and M. Steck, Proceedings of EPAC 1992, p. 123.

[5] S. Chattopadhyay, CERN Report No. 84-11, 1984.

[6] V. V. Parkhomchuk and D. V. Pestrikov, Sov. Phys. Tech. Phys. 50, 1411 (1980).

[7] D. V. Pestrikov, Nucl. Instrum. Methods Phys. Res., Sect. A 562, 65 (2006).

[8] D. V. Pestrikov, Nucl. Instrum. Methods Phys. Res., Sect. A 578, 65 (2007).
[9] V. Kornilov, O. Boine-Frankenheim, and I. Hofmann, Phys. Rev. ST Accel. Beams 11, 014201 (2008).

[10] K. Blasche and B. Franczak, Proceedings of EPAC 1992, p. 9.

[11] O. Boine-Frankenheim and V. Kornilov, Proceedings of ICAP2006, Chamonix Mont-Blanc, 2006.

[12] I. Hofmann and G. Kalisch, Phys. Rev. E 53, 2807 (1996).

[13] O. Boine-Frankenheim and T. Shukla, Phys. Rev. ST Accel. Beams 8, 034201 (2005).

[14] K. Y. Ng, Report No. FERMILAB-FN-0760-AD, 2004.

[15] K.Y. Ng, Physics of Intensity Dependent Beam Instabilities (World Scientific, Singapore, 2006).

[16] M. Reiser, Theory and Design of Charged Particle Beams (Wiley, New York, 1994).

[17] C. K. Birdsall and A. Langdon, Plasma Physics via Computer Simulation (IOP, Bristol, 1991).

[18] T. Giacomini, S. Barabin, P. Forck, D. Liakin, and V. Skachkov, AIP Conf. Proc. 732, 286 (2004). 